\title{
WDM Supervision in PHOTON as a Basis for OAM of All-Optical Transport Networks
}

\author{
Michael Lehdorfer, Siemens AG Austria, Vienna \\ Oliver Jahreis, Siemens AG, Munich
}

Correspondence Address:

Michael Lehdorfer

Siemens AG Austria, PSE EZE TNT 4

A-1031 Wien, Erdberger Lände 26

EMail: michael.lehdorfer@siemens.at

Phone: +43-1-1707-35129

Fax : +43-1-1707-55120

\section{Abstract}

A key issue for the Operation \& Maintenance (OAM) of an all-optical transport network is failure localisation in the network. The WDM supervision concept in the ACTS project PHOTON (Photonic Transport Overlay Network) achieves this by the introduction of defects and maintenance signals similar to existing transport networks. In the following this concept is explained.

This work was supported in part by the European Commission. The contents of this paper is solely the responsibility of the authors. 


\section{The used Layer Concept}

For the purpose of defining OAM principles a layered model for the optical transport network is very useful. Within PHOTON, which utilises Wavelength Division Multiplexing (WDM), such a model has been defined, based on the generic architecture of the ITU G.805 recommendation, by introducing three new layers between the existing physical media layer and the existing transport layer networks (e.g. SDH or ATM). The new proposal of G.otn for optical transport networks has not been taken into account, since it was not available at the time the PHOTON model had been established and G.otn currently is not more than a draft. Nevertheless the standardisation process within G.otn will be observed carefully. The G.lon, now already agreed as G.681, on the other hand, deals only with WDM point-to-point connections supporting SDH and cannot be applied directly to all-optical transport networks.

The three layers for the optical transport network are:

- Client regenerator section layer

- Optical path layer

- Optical frequency section layer

- Optical regenerator section layer

- Physical media layer

Figure 1 shows the corresponding sections in the network. The optical path can be seen as a transport pipe through the optical transport network. The optical frequency section extends between the optical cross connects (OCCs), and the optical regenerator section is terminated by optical regenerators (ORs) or optical nodes. 


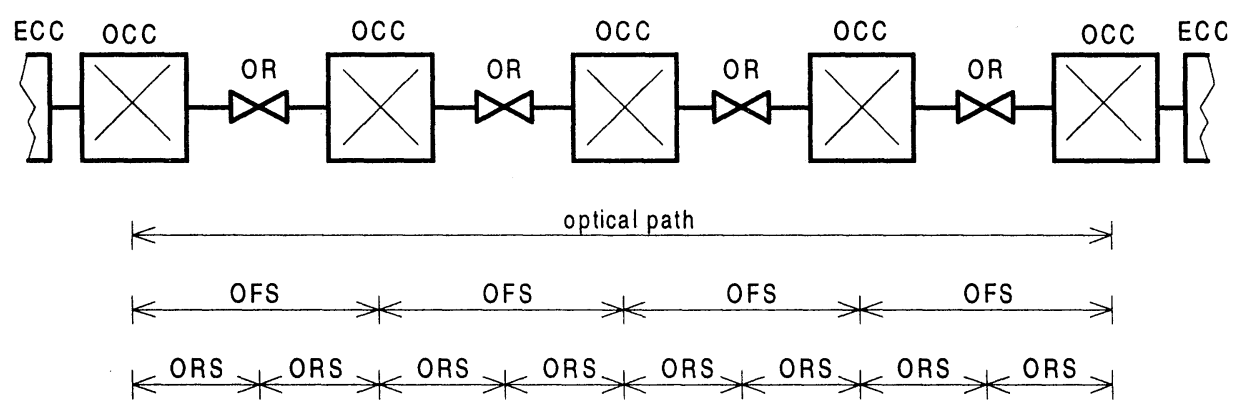

OFS Optical frequency section OR Optical regenerator

OCC Optical cross connect ECC Electrical cross connect

Figure 1: Optical path and optical sections in optical transport networks

Each of the three optical layers has (at least logically) an overhead assigned which carries WDM supervision information. These layer overheads have the same purpose as the overheads in the SDH transport networks and represent the payload independent information in the optical transport network. This information is used for network internal purposes only. In the PHOTON optical transport network it is proposed to use special WDM channels (so called overhead channels) reserved only for the purpose of overhead transmission and network management information transport within the optical transport network. Maintenance signals are part of the layer overheads and transported within the overhead channels to enable failure detection, failure localisation and failure suppression inside the optical transport network. The generation of these signals is strongly related to the defects detected by the optical nodes in the optical transport network. 


\section{Defects and Maintenance Signals}

Figure 2 lists the defects and maintenance signals for each of the three optical layers. Here it is assumed that all three overheads are transported in one overhead channel.

\begin{tabular}{|l|l|l|l|}
\cline { 2 - 4 } \multicolumn{1}{c|}{} & $\begin{array}{l}\text { Optical regenerator } \\
\text { section layer }\end{array}$ & $\begin{array}{l}\text { Optical frequency } \\
\text { section layer }\end{array}$ & $\begin{array}{l}\text { Optical path } \\
\text { layer }\end{array}$ \\
\hline \multirow{4}{*}{$\begin{array}{l}\text { Main- } \\
\text { tenance } \\
\text { signals }\end{array}$} & $\begin{array}{l}\text { ORS-alarm indication } \\
\text { signal }\end{array}$ & OFS-remote defect indication & $\begin{array}{l}\text { OP-alarm indication } \\
\text { signal }\end{array}$ \\
\cline { 2 - 5 } & OH failure indication signal & Channel defect indication & $\begin{array}{l}\text { OP-remote defect } \\
\text { indication }\end{array}$ \\
\cline { 2 - 5 } & - & Frequency shift indication & - \\
\hline \multirow{4}{*}{$\begin{array}{l}\text { Detected } \\
\text { defects }\end{array}$} & Loss of signal & Unequipped signal & - \\
\cline { 2 - 5 } & Loss of overhead & Loss of channel & $\begin{array}{l}\text { Path identifier } \\
\text { mismatch }\end{array}$ \\
\cline { 2 - 5 } & Shift of frequency & $\begin{array}{l}\text { Loss of tributary } \\
\text { channel signal }\end{array}$ \\
\hline
\end{tabular}

Figure 2: Defects and maintenance signals 
In the following each defect and maintenance signal is described shortly:

\section{Defects}

- Loss of signal (LOS): the complete WDM signal fails.

- Loss of payload (LOP): all payload channels fail at the same time and the overhead is still present.

- Loss of overhead (LOH): the overhead fails.

- Loss of channel (LOC): a single payload channel fails.

- Channel degrade (CD):the signal quality of a single channel is below a certain level.

- Shift of frequency (SOF): the frequency of a single channel is out of range.

- Path identifier mismatch (PID): an optical path signal is not the one expected at the path end.

- Loss of tributary channel (LTC): an optical single channel signal, which is provided to the optical transport network by the client network, fails.

- Degradation of tributary channel (DTC): the signal quality of an optical single channel signal which is provided to the optical transport network by the client network is below a certain range.

\section{Maintenance signals}

- ORS - alarm indication signal (ORS-AIS): informs all downstream network elements along an OFS about LOS or LOP.

- OFS - remote defect indication (OFS-RDI): informs the next upstream optical node about LOS or LOP.

- Channel defect indication (CDI): informs the next upstream optical node about LOC or CD.

- Frequency shift indication (FSI): informs the next upstream optical node about SOF. 
- Overhead failure indication signal (OHFIS): informs all downstream network elements along and OFS about faulty overhead.

- Unequipped signal (UNEQ): informs the downstream optical nodes about the intentional unequipped state of a payload channel.

- OP - alarm indication signal (OP-AIS): informs the downstream optical nodes about a faulty payload channel.

- OP - remote defect indication (OP-RDI) informs the upstream optical nodes about a faulty payload channel

\section{Maintenance Signal Interaction}

In this section the maintenance signals and their interaction are presented with the simple example depicted in figure 3. A fibre break between an optical cross connect and an optical regenerator is assumed. This network failure results in the detection of the LOS defect in the optical regenerator directly behind this break. As a result ORS-AIS is generated in downstream direction. This signal informs the OCC about the failure and suppresses its defect detection for this fibre. Additional the OHFIS is generated by the OR, informing the OCC about the loss of the overhead and the location of the reporting OR. The OCC reacts with generation of additional maintenance signals. An OFS-RDI signal informs the upstream OCC about the failure of the OFS and enables e.g. to stop billing for this line. In downstream direction the OCC generates an OP-AIS for all optical path affected by this fibre break. This signal suppresses the defect detection for these paths (e.g. LOC) in the downstream OCCs. An OP-RDI is generated at the end of the optical path to inform the other path end about the defective connection. 

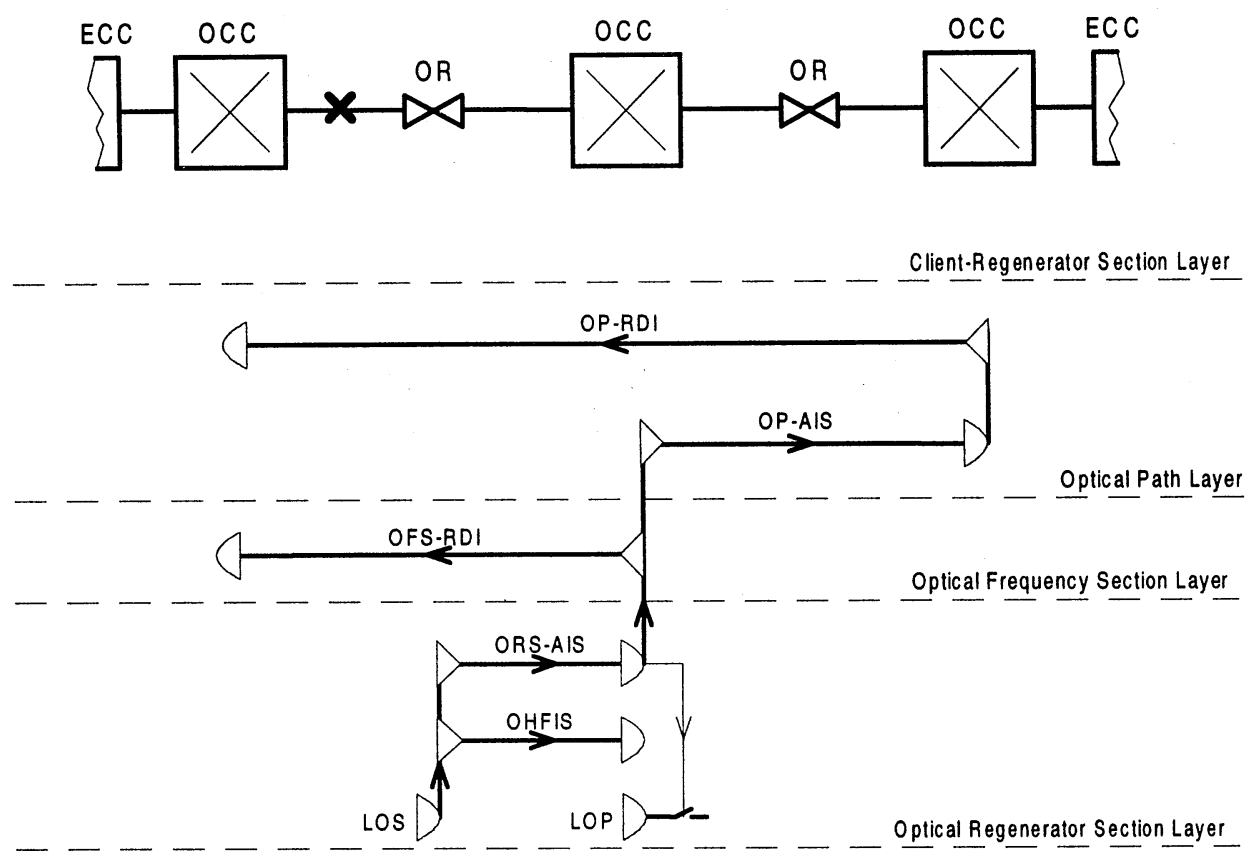

$\gg$ signal generation

Detection

Fibre break

Figure 3: Maintenance signal flow and interaction

With the use of these signals and defect definitions it is not only possible to localise the failure in the optical transport network. But this information can also be used for protection mechanisms in the network which enhance the optical transport network availability.

\section{Conclusions and Future Work}

A means to enable failure localisation in the all-optical transport network is presented. This WDM supervision concept will be real- 
ised within the ACTS projects PHOTON and MOON (Management Of Optical Networks) field trial. The MOON field trial will extend the PHOTON field trial with the appropriate network management which contributes to the aim of a complete OAM for optical transport networks.

But still work on the field of an OAM for optical transport networks has to be done. The presented concept concentrates on the supervision of the optical sections and the optical path. Extending supervision also to the optical nodes will lead to a comprehensive OAM concept for future all optical networks. 